\title{
Chirped dissipative solitons of the complex cubic-quintic nonlinear Ginzburg-Landau equation
}

\author{
V. L. Kalashnikov \\ Institut für Photonik, TU Wien, Gusshausstr. 27/387, A-1040 Vienna, Austrid*
}

\begin{abstract}
Approximate analytical chirped solitary pulse (chirped dissipative soliton) solutions of the onedimensional complex cubic-quintic nonlinear Ginzburg-Landau equation are obtained. These solutions are stable and highly-accurate under condition of domination of a normal dispersion over a spectral dissipation. The parametric space of the solitons is three-dimensional, that makes theirs to be easily traceable within a whole range of the equation parameters. Scaling properties of the chirped dissipative solitons are highly interesting for applications in the field of high-energy ultrafast laser physics.
\end{abstract}

PACS numbers: 42.65.Tg, 42.65.Re

*Electronic address: kalashnikov@tuwien.ac.at 


\section{INTRODUCTION}

The complex nonlinear Ginzburg-Landau equation (CGLE) has so wide scope of applications that the concept of "the world of the Ginzburg-Landau equation" [1] is not exaggeration. The CGLE demonstrates its effectiveness in quantum optics, modeling of Bose-Einstein condensation, condensate-matter physics, study of non-equilibrium phenomena, and nonlinear dynamics, quantum mechanics of self-organizing dissipative systems, and quantum field theory. In optics and laser physics, the CGLE provides an adequate description of modelocked oscillators and pulse propagation in fibers [2, 3].

The CGLE is multiparameter and not integrable in a general form. As a result, an analysis of multitude of its solutions requires extensive numerical simulations. The exact analytical solutions are known only for a few of cases, when they represent the solitary waves (dissipative solitons) and some algebraic relations on the parameters of equation are imposed [2, 4]. As a rule, one presumes some class of functional expressions to construct the solution. As a result of such presupposition, the solutions outside a given class are missed. In principle, the missed solutions can be revealed on basis of the algebraic non-perturbative techniques [4, 5], which, nevertheless, need a lot of computer algebra. These challenges stimulate interest in the approximate methods of integration of the CGLE.

The perturbative method has allowed obtaining the dissipative soliton solutions of the reduced and complete cubic-quintic CGLE in the limits of small and large dispersion and phase nonlinearity [6, 7]. Another approximate approach is to reduce an infinite-dimensional (in terms of degrees of freedom) problem to finite-dimensional one on basis of, for instance, the method of moments. This allows tracing an evolution of a finite set of the trial solution parameters [8].

However, some physically interesting sectors of the CGLE allow an approximation without any functional constraints imposed on the solution or/and the equation parameters. Moreover, dimensionality of the parametric space corresponding to such a solution can be reduced in comparison with the parametric space of the CGLE that makes the solution under consideration to be easily traceable.

A physically important sector, which permits an approximate analysis, is represented by the chirped solitary pulse solutions, or the chirped dissipative solitons (CDSs) of the CGLE. The CDS exists in both anomalous and normal dispersion ranges [8, 9, 10]. It is 
very important, that the CDS is energy scalable [8, 10, 11, 12] and can be considered as a model of femtosecond laser pulses with about of and over microjoule energies [13, 14]. Energy scalability of the CDS results from its stretching caused by a large chirp. Hence, the CDS with large energy has a reduced peak power that provides its stability [15, 16]. Simultaneously, a large chirp leads to spectral extra-broadening so that the CDS becomes to be compressible down to a few of tens of femtoseconds [11, 13, 15].

The mechanism of the CDS formation is a composite balance of phase and dissipative effects [8]. The first effect is a balance of phase contributions from the nonlinear phase distortion and the time-dependent phase affected by a normal dispersion [17]. That is possible if a soliton is chirped, but this effect alone does not provide a soliton stability. The CDS stability can be provided by a balance between the nonlinear gain and the spectral dissipation [9, 17, 18].

A large chirp of the CDS allows two main approximations: i) soliton stretching admits the adiabatic approximation, ii) fast phase variation allows applying the stationary phase method in Fourier domain [15, 16, 20]. As a result, the CDS of the reduced cubic-quintic onedimensional CGLE [15, 20] and the generalized one-dimensional CGLE (i.e. the CGLE with an imaginary saturable law nonlinearity in terms of [19]) [16] can be represented analytically as the two-parametric solitary pulse solution without any restrictions on its functional form as well as on the equation parameters (certainly, within the scope of approximations under consideration, see below).

Here the extension of this approximate technique to the complete cubic-quintic onedimensional CGLE (i.e. the CGLE with a complex parabolic law nonlinearity [19]) is presented. It is shown, that the CDS is the three-parametric solution with five types of the truncated spectral profiles: i) finger-, ii) parabolic-, and iii) flat-top, as well as iv) concave and v) concave-convex ones. The regions of existence and stability of the CDS are analyzed systematically within a whole parametric range obeying the condition of domination of a normal dispersion over a spectral dissipation. The obtained results are validated on the basis of numerical solution of the CGLE and compared with the existing results of extensive numerical simulations of the CGLE. 


\section{CDS OF THE CUBIC-QUINTIC CGLE}

Let the CGLE be written down in the following form [15, 20]:

$\frac{\partial}{\partial z} a(z, t)=-\sigma a(z, t)+(\alpha+i \beta) \frac{\partial^{2}}{\partial t^{2}} a(z, t)+(\kappa-i \gamma)|a(z, t)|^{2} a(z, t)-(\kappa \zeta+i \chi)|a(z, t)|^{4} a(z, t)$.

Here $z$ is the propagation (longitudinal) coordinate, which can be the propagation distance in a fiber, or the cavity round-trip in a laser oscillator, for instance; $t$ is the "transverse" coordinate, which can be, for instance, the local time for a propagating laser pulse [2]. The complex slowly-varying field amplitude $a(z, t)$ is chosen so that $|a|^{2}$ has a dimension of instant power. The first term on right-hand side of Eq. (11) describes an action of net-loss with the parameter $\sigma$. In the general case, this parameter is energy-dependent (i.e. it depends on $\int_{-\infty}^{\infty}\left|a\left(z, t^{\prime}\right)\right|^{2} d t^{\prime}$, see [15]) and has to be positive to provide the vacuum stability [ i.e. the subcritical range of Eq. (11) is under consideration]. The second term describes a spectral dissipation ( $\alpha$ is the squared inverse bandwidth of spectral filter) and a dispersion $[\beta$ is the dispersion coefficient]. Positivity (negativity) of $\beta$ corresponds to normal (anomalous) dispersion. The third term results from a contribution of cubic nonlinearity, which is a sum of contributions from the nonlinear gain [or the self-amplitude modulation (SAM) defined by the parameter $\kappa>0$ ] and from the self-phase modulation (SPM) with the parameter $\gamma$. Only the focusing SPM with $\gamma>0$ will be considered below. A higher-order (quintic) nonlinearity defines the fourth term in Eq. (1). Its real part describes the SAM saturation ( $\zeta>0$ provides stability of a desired solution against collapse), while a correction to the cubic SPM can be both enhancing $(\chi>0)$ and saturating $(\chi<0)$.

To find the CDS solution, let's make the traveling wave reduction of Eq.(1) by means of the ansatz

$$
a(z, t)=\sqrt{P(t)} \exp [i \phi(t)-i q z]
$$

where $P(t)$ is the instant power, which defines a CDS envelope; $\phi(t)$ is the phase, and $q$ is the phase shift due to a slip of the carrier phase with respect to an envelope [3].

Below we shall consider only the sector of CGLE, where a normal dispersion prevails over a spectral dissipation, that is $\beta \gg \alpha>0[6]$. This assumption is well-grounded for both 
broadband solid-state [11, 15, 16] and fiber [16, 21, 22] laser oscillators operating in the all-normal dispersion (ANDi) regime. But the numerical analysis demonstrates [16], that even the case of $\beta \geq \alpha>0$ (e.g., a thin-disk solid-state oscillator [14]) can be described adequately in the framework of the analytical approach under consideration.

The adiabatic approximation $T \gg \sqrt{\beta}$ allows obtaining from Eqs. (12)

$$
\begin{aligned}
& \beta \Omega^{2}=q-\gamma P-\chi P^{2}, \\
& \beta\left(\frac{\Omega}{P(t)} \frac{d P}{d t}+\frac{d \Omega}{d t}\right)=\kappa P(1-\zeta P)-\sigma-\alpha \Omega^{2},
\end{aligned}
$$

where $\Omega \equiv d \phi(t) / d t$ is the instant frequency.

Since the first equation in (3) is quadratic in $P$, there are two branches of solution. However, it is reasonable to confine oneself to the branch, which has the limit $\chi \rightarrow 0$ (this limit has been considered in [15, 20]). Then, one has

$$
P=-\frac{1}{2 \chi}\left(\gamma-\sqrt{\gamma^{2}+4 q \chi-4 \chi \beta \Omega^{2}}\right)
$$

Since $P \geq 0$ by definition, there is the maximum frequency deviation $\Delta$ from the carrier frequency: $\Delta^{2}=q / \beta$. Thus, the second equation in (3) and Eq. (44) lead, after some algebra [23], to

$$
\begin{aligned}
& \frac{d \Omega}{d t}=\frac{\sigma+\alpha \Omega^{2}-\frac{\kappa(A-\gamma)}{4 \chi^{2}}(2 \chi+\zeta \gamma-\zeta A)}{\beta\left[4 \chi \beta \Omega^{2}-(A-\gamma) A\right]}(A-\gamma) A, \\
& A=\sqrt{\gamma^{2}+4 \beta \chi\left(\Delta^{2}-\Omega^{2}\right)} .
\end{aligned}
$$

The singularity points of Eq. (5) impose the restrictions on the $\Delta$ value [23]

$\Delta^{2}=\frac{\gamma}{16 \zeta \beta\left(\frac{c}{b}+1\right)}\left[\frac{2\left(3+\frac{c}{b}+\frac{4}{b}\right)\left(2+\frac{c}{2}+\frac{3 b}{2} \pm \sqrt{(c-2)^{2}-16 a\left(1+\frac{c}{b}\right)}\right)}{1+\frac{c}{b}}-12-3 c-9 b-\frac{32 a}{b}\right]$

where three control parameters are $a \equiv \sigma \zeta / \kappa, b \equiv \zeta \gamma / \chi$, and $c \equiv \alpha \gamma / \beta \kappa$. These three parameters define the parametric dimensionality of the CDS. Eqs. (4, [6] allow obtaining the CDS peak power ( $\Omega$ has to be equal to 0 in Eq. (4) for this aim). 
It is convenient to use the following normalizations: $t^{\prime}=t(\kappa / \zeta) \sqrt{\kappa / \alpha \zeta}, \Delta^{\prime 2}=\Delta^{2} \alpha \zeta / \kappa$, $\Omega^{\prime 2}=\Omega^{2} \alpha \zeta / \kappa, P^{\prime}=\zeta P$. For the dimensionless energy, one has $E^{\prime}=E(\kappa / \gamma) \sqrt{\kappa \zeta / \alpha}$. Hereinafter, these normalization will be implied and the primes will be omitted.

The expressions for the dimensionless quantities are

$$
\begin{aligned}
& A=\sqrt{1+\frac{4\left(\Delta^{2}-\Omega^{2}\right)}{c b},} \\
& P=\frac{b}{2}\left(\sqrt{1+\frac{4\left(\Delta^{2}-\Omega^{2}\right)}{c b}}-1\right), \\
& \frac{d \Omega}{d t}=\frac{c\left[a+\Omega^{2}+\frac{b^{2}}{4}(1-A)\left(\frac{2}{b}+1-A\right)\right] A(A-1)}{\frac{4 \Omega^{2}}{c b}+A(1-A)}, \\
& \Delta^{2}=\frac{c}{16\left(\frac{c}{b}+1\right)}\left[\frac{2\left(\frac{c}{b}+3+\frac{4}{b}\right)\left(\frac{c}{2}+\frac{3 b}{2}+2 \pm \sqrt{(c-2)^{2}-16 a\left(\frac{c}{b}+1\right)}\right)}{\frac{c}{b}+1}-3 c-9 b-\frac{32 a}{b}-12\right] .
\end{aligned}
$$

Since the phase $\phi(t)$ of the CDS can be treated as a rapidly varying function of time in the limit of $\kappa \ll \gamma$, one may apply the method of stationary phase to the Fourier image of $a(t)[20]$. As a result, the expression for the CDS spectral profile is

$$
p(\omega) \equiv|e(\omega)|^{2} \approx \frac{\pi(A-1)\left((A-1) c b+4\left(2 \omega^{2}-\Delta^{2}\right)\right) H\left(\Delta^{2}-\omega^{2}\right)}{c A\left((A-1)\left(c\left(a+b+b^{2}+\omega^{2}\right)+b\left(\Delta^{2}-\omega^{2}\right)\right)-2(b+1)\left(\Delta^{2}-\omega^{2}\right)\right)} .
$$

where $e(\omega) \equiv \int d t \sqrt{P(t)} \exp [i \phi(t)-i \omega t], H(x)$ is the Heaviside's function and one has to replace $\Omega$ by $\omega$ in $A$ given by Eq. (7).

The CDS energy can be obtained from Eq. (8) by integration: $E=\int_{-\Delta}^{\Delta} \frac{d \omega}{2 \pi} p(\omega)$. This value can be related to the energy $E^{*}$ of a solution of the linearized version of Eq. (11) through the saturated net-loss parameter $\sigma: \sigma \approx \delta\left(E / E^{*}-1\right)$, where $\delta \equiv d \sigma /\left.d E\right|_{E=E^{*}}$ [15]. Such a relation can be usable, for instance, to define the CDS parameters from those of a laser oscillator [15, 16].

Thus, the approximate technique under consideration allows representing the CDS parameters, its spectral and temporal profiles as well as energy from a few of algebraic expressions (7],8), single first-order ordinary differential equation (7) and numerical integration of (8). Since the CDS is three-parametric, such an approximation allows easily tracing the soliton 
characteristics within a broad range of the CGLE parameters. It is important, that the absolute values of the CGLE parameters are not relevant in contrast to their relations presented by the parameters $a, b$ and $c$. This allows a unified viewpoint at the diverse systems obeying the CGLE [16, 24].

\section{MASTER DIAGRAM AND REGIONS OF THE CDS EXISTENCE}

Eq. (17) demonstrates that there exist two branches of the CDS corresponding to two signs before square root in the expression for $\Delta$. As will be shown below, such a division into two branches is physically meaningful. In accordance with the sign in Eq. (7), we shall denote these branches as the "positive" $(+)$ and "negative" $(-)$ ones. One has note, that only the - branch has a limit for $\zeta, \chi \rightarrow 0$ (the "Schrödinger limit") [20].

\section{A. Positive branch of the CDS}

Regions of the + branch existence are shown in Fig. 11 on the plane $(a-c)$ for the different $b$. Zero value of $a$ corresponds to a marginally stable CDS. The existence regions are maximally broad in this case. The restrictions on the parameters are $0<c<2$, and $b>0$ (the SPM is unsaturable) or $b<-c / 3-4 / 3$ (the SPM is saturable). One has to resemble, that the decrease of $|b|$ means a growth of contribution of the quintic SPM. The physical meaning of maximum $c$ is that there is a minimum dispersion or a maximum spectral dissipation, which provides the CDS existence.

A new view on the CDS results from a consideration of chirp at the soliton center (i.e. at $t=0)$ :

$$
\begin{aligned}
\left.\psi\right|_{t=0} \equiv & \left.\frac{d \Omega}{d t}\right|_{\Omega=0}=-a-\frac{b^{2}}{4}\left(1-\left.A\right|_{\Omega=0}\right) \\
& \times\left(1+\frac{2}{b}-\left.A\right|_{\Omega=0}\right) .
\end{aligned}
$$

Here the chirp is normalized to $\kappa / \zeta \beta$. The analysis demonstrates that the chirp becomes negative, when the $c$ parameter reaches some minimum value (the lower borders of the hatched regions in Fig. 11). Such a chirp corresponds to a spike on constant background with $\lim _{t \rightarrow \pm \infty} \Omega< \pm \infty, \lim _{t \rightarrow \pm \infty} P=$ const $>0$. These solutions will be not considered hereafter 


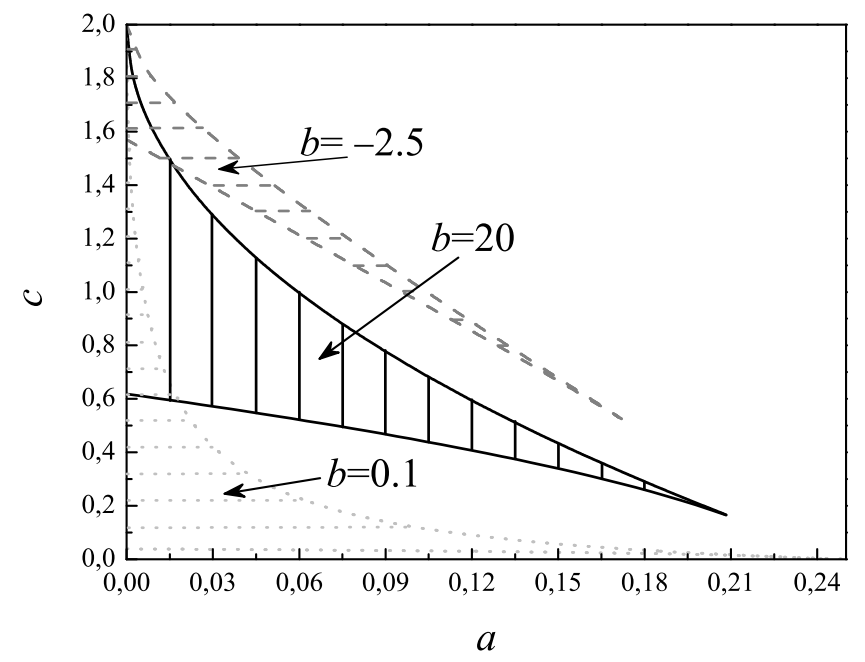

FIG. 1: Regions (hatched) of the + branch existence. $b=20$ (black solid curves, vertical hatching), 0.1 (light gray dotted curves, horizontal hatching), and -2.5 (gray dashed curves, horizontal hatching).

and the chirp positivity will be admitted as the additional criterion of the CDS existence. This criterion agrees with the analytical results of [10] in the limit of $\alpha / \beta \ll 1$ and $\kappa / \gamma \ll 1$. The appropriate zero asymptotic $\lim _{t \rightarrow \pm \infty} P=0$ of the solutions analyzed in [10] exists only if $0<\psi \approx 3 \gamma /(1+c) \kappa<\beta / \alpha$. Here $\psi$ is defined as the parameter in the phase profile ansatz $\phi(t)=\psi \ln \sqrt{P(t)}$, which is used in [10].

As a result, there is some minimum $c$ (i.e. maximum normal dispersion or minimum spectral dissipation) for the + branch (Fig. 1). This minimum $c$ tends to zero, when the positive $b$ decreases (Fig. 1).

For $b<0$, the CDS existence range squeezes, when $b$ approaches -2 (Fig. 11). If $b>-2$, the positively chirped CDS has a parabolic temporal profile and $\lim _{t \rightarrow \pm \tau} \Omega= \pm \infty, \lim _{t \rightarrow \pm \tau} P=0$ ( $\tau$ is some finite interval of local time). We will not consider such an "inverted" CDS hereafter.

The $a$ growth, if it results from the $\sigma$ increase, enhances the soliton stability against a vacuum destabilization. However, the existence regions shrink along the $c$ parametric coordinate with such a growth (Fig. 1). 


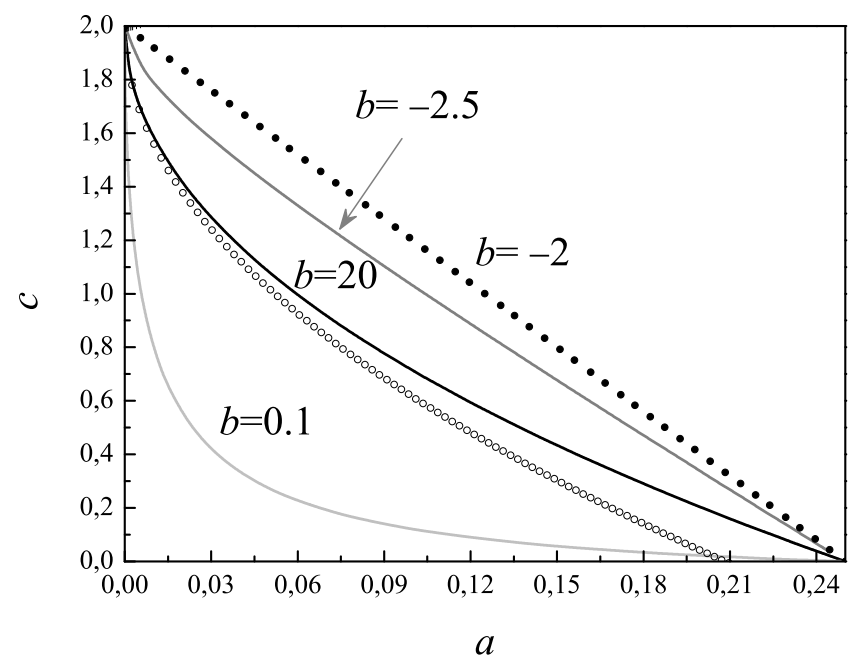

FIG. 2: Borders of regions of the - branch existence (black solid curve for $b=20$, light gray solid curve for $b=0.1$, gray solid curve for $b=-2.5$, points for $b=-2$ ). Open circles correspond to the region border from [6]. The existence regions lie below the corresponding borders.

\section{B. Negative branch of the CDS}

Regions of the - branch existence are shown in Fig. 2 on the plane $(a-c)$ for the different b. The CDS exists within the interval $0<c<2$, which squeezes with $a$. Since this branch has a Schrödinger limit, such a squeezing can be obtained on the basis of the perturbative method [6]. Then, the existence region for $|b| \gg 1$ is $c \leqslant 2-4 \sqrt{6 a / 5}$ (open circles in Fig. 21) [6]. One can see, that the approximation of [6] is quite accurate, when $a \ll 1$ (i.e. in the low-energy limit). The limiting $a$ is defined by the hard excitation condition $a \leqslant 1 / 4[6]$. The existence region shrinks with a growing positive quintic SPM (i.e., when $b>0$ tends to zero) and stretches with a negative quintic SPM verging towards $b=-2$.

There are no negative chirp solutions for this branch. There exist the positive chirp solutions for $c>2$ and $b<-2$, but they are the spikes on background.

One can see that the upper (in the $c$ direction) borders of the regions coincide for the positive and negative branches. This means that the branches coexist within the regions of their existence in the $(a, b, c)$-parametric space. 


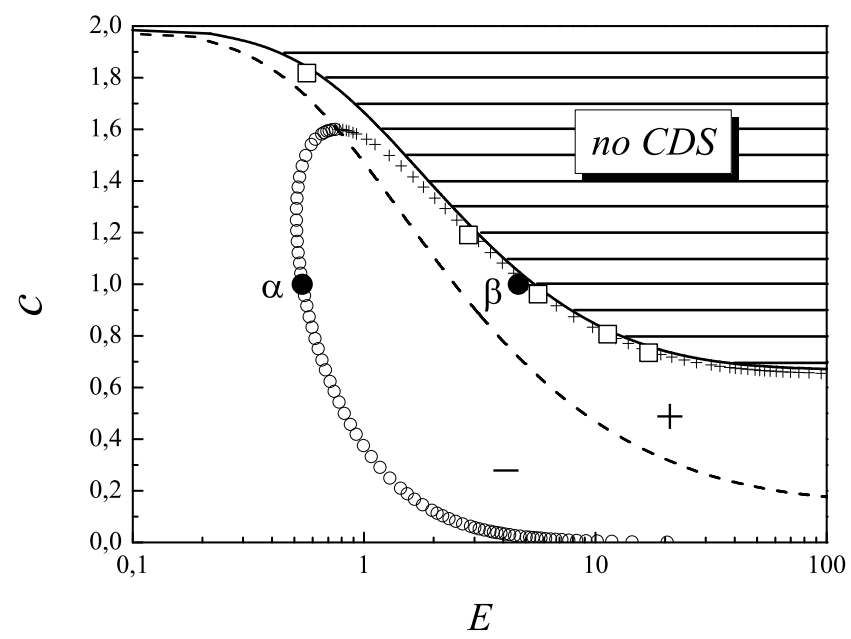

FIG. 3: The master diagram for $b \gg 1$. There exists no CDS within the hatched region. Solid curve corresponds to $a=0$. Dashed curve divides the regions, where the + and - branches exist. Crosses (circles) correspond to the $+(-)$ branch for $a=0.01$. The points $\alpha$ and $\beta$ correspond to the parameters of the numerical solutions presented in Figs. 89 by open circles. The open squares indicate the numerically obtained stability border $(\kappa=0.04 \gamma, \zeta=0.5 \gamma)$.

\section{Master diagram}

Representation of the existence regions in the form of Figs. 112 is awkward in some way, because the $a$ parameter can be energy-dependent. As a result, the branches do not coexist as they differ in energy. It is more convenient to use a representation on the plane $(E-c)$ for the different $b$. Such a representation will be called the master diagram. The $E$ value can be easily related to the experimentally controllable parameter $E^{*}$ (see Section II).

The master diagram for the CDS is shown in Fig. 3 for the case of vanishing quintic SPM $(b \gg 1[15,24])$. The solid curve shows the border of the CDS existence $(a=0)$. Above this border, the vacuum of Eq. (1) is unstable (hatched region). The dashed curve divides the existence regions for the + and - branches (the branches merge along this curve). Crosses (circles) represent the curve along which there exists the $+(-)$ branch for some fixed value of $a$ (so-called the isogain curve).

The master diagram is interrelated with the existence regions in Figs. 1,2, The point of intersection of isogain with the dashed curve defines the maximum value of $c$ in Figs. 1,2 for the corresponding $a$. Since the + branch isogain has a nonzero asymptotic for $E \rightarrow \infty$, there is the nonzero minimum $c$, which confines the + branch region for a fixed $a$ in Fig. 
1. The - branch has a zero asymptotic for $E \rightarrow \infty$. Hence, the - branch extends down to $c=0$.

The master diagram reveals four significant differences between the branches. The first one is that the - branch has lower energy than the + branch for a fixed $c$. The second difference is that the + branch isogain has nonzero asymptotic for $E \rightarrow \infty$. In this sense, the + branch is energy scalable, that is its energy growth does not require a substantial change of $c$. The - branch is not energy scalable, that is its energy growth needs a substantial decrease of $c$ (e.g., owing to a dispersion growth) [16]. The third difference is that the + branch verges on $\sigma=0$ within a whole range of $E$. The fourth difference is that the - branch has a Schrödinger limit $\zeta, \chi \rightarrow 0$.

Growth of the positive quintic SPM (i.e. $b \rightarrow 0$ ) narrows the existence region (Fig. 4). This means that smaller $c$ is required to provide the CDS existence for some $E$. That is, since the positive quintic SPM means an enhancement of the phase nonlinearity, a SPM enhancement has to be compensated, for instance, by a dispersion increase $(c \propto 1 / \beta)$. One can see from Fig. 4 , that the + branch region narrows substantially with $b \rightarrow 0(b>0)$ within a whole range of $E$.

The situation is opposite, when the quintic SPM is negative. The existence range widens and a larger $c$ (i.e. smaller dispersion) provides the CDS existence for some $E$. The + branch region widens, as well. However, it is important to remember, that the range of $c$, where the CDS with a fixed $a$ exists, is defined by the difference between i) the point of intersection of the isogain with the boundary between the + and - branches and ii) the isogain asymptotic for $E \rightarrow \infty$. As a result, the range of $c$, where some isogain exists, can be narrow in spite of the fact that a whole range of the + branch widens (Figs. 10.5). The reversed situation, when a whole existence range is narrow, but the range of $c$ for some isogain is broad, is possible for $b>0$ (Figs. 104).

\section{CDS PROFILE, SPECTRUM AND PARAMETERS}

Fig. 6] shows the frequency deviations and the CDS profiles relating to the + branch [see Eq. (7)] for the different $b$. One can see, that the decrease of positive $b$ reduces a soliton energy (black solid vs. gray curves in Fig. 6i) for the fixed $c$ and $a$. That agrees with Figs. 34, where the isogain shifts towards smaller energies for a fixed $c$, when the positive $b$ tends 


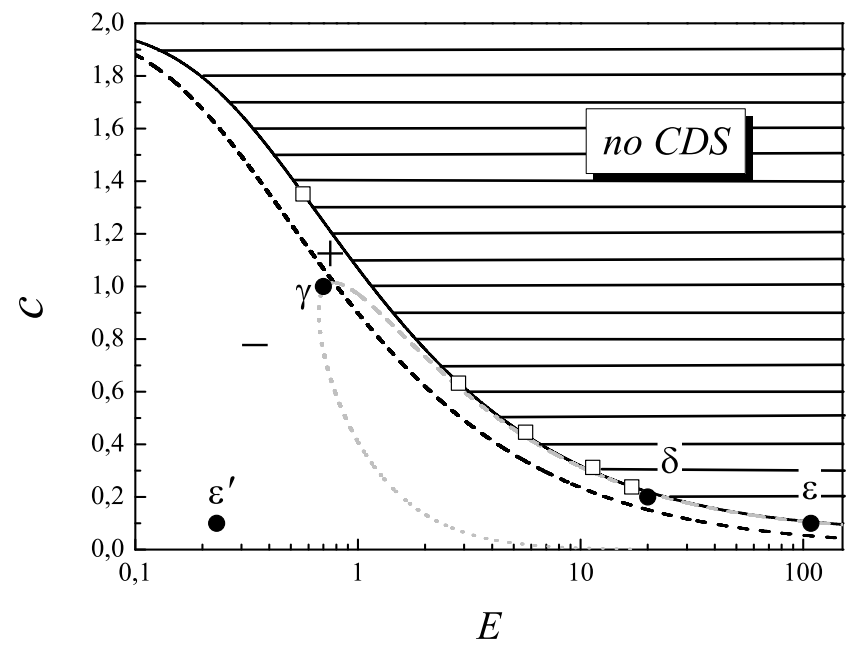

FIG. 4: The master diagram for $b=0.2$. There exists no CDS within the hatched region. Black solid curve corresponds to $a=0$. Black dashed curve divides the regions, where the + and - branches exist. Gray dashed (dotted) curve corresponds to the $+(-)$ branch for $a=0.01$. The point $\gamma$ corresponds to the parameters of the numerical solution presented in Fig. 9 by open squares. The open squares indicate the numerically obtained stability border $(\kappa=0.04 \gamma, \zeta=0.5 \gamma)$. The points $\delta$ and $\varepsilon$ correspond to the analytical spectra presented in Fig. $12(\kappa=0.8 \gamma$ and $1.5 \gamma$, respectively; $\beta / \alpha=6.25, \zeta=0.002 \gamma, \sigma=0.1[12])$. The point $\varepsilon^{\prime}$ is the - branch counterpart of $\varepsilon$.

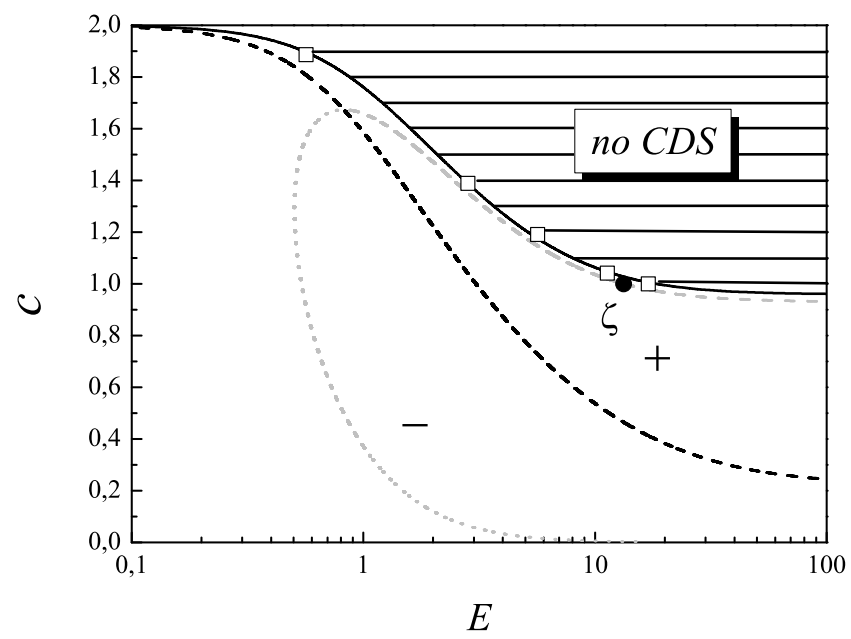

FIG. 5: The master diagram for $b=-5$. There exists no CDS within the hatched region. Black solid curve corresponds to $a=0$. Black dashed curve divides the regions, where the + and - branches exist. Gray dashed (dotted) curve corresponds to the $+(-)$ branch for $a=0.01$. The point $\zeta$ corresponds to the parameters of the numerical solution presented in Fig. 8 by open squares. The open squares indicate the numerically obtained stability border $(\kappa=0.04 \gamma, \zeta=0.5 \gamma)$. 


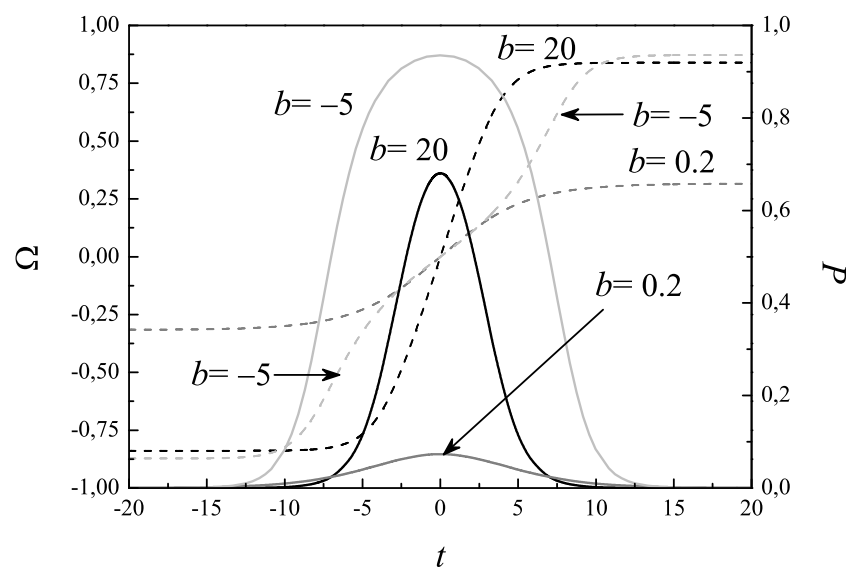

FIG. 6: The + branch CDS profiles (solid curves) and frequency deviations (dashed curves) for the different $b . c=1, a=0.01$.

to zero. Since a power decreases, a chirp $(d \Omega / d t)$ decreases, too (black vs. gray dashed curves in Fig. 6).

In the case of $b<0$, the dependence of $\Omega$ on $t$ becomes "loitering" (light gray dashed curve in Fig. 6). As a consequence, the CDS profile becomes flat-top (light gray solid curve in Fig. 6). The energy increases for a fixed $a$ in agreement with a shift of the isogain towards larger energies in Fig. 5 .

The frequency deviations and the CDS profiles for the - branch are shown in Fig. 7, Out of the boundary between the branches, the CDS relating to the - branch has lower energy and power than its + counterpart. Correspondingly, a chirp is lower, as well. Growth of the positive quintic SPM (i.e., $b \rightarrow 0$ ) increases the soliton energy, power and chirp (black vs. gray curves in Fig. 7).

Growth of the negative quintic SPM decreases the CDS energy, power and chirp. However, such a decrease is comparatively small and, therefore, it is not shown in Fig. 7.

The CDS spectra are presented in Figs. 8,9, As has been shown in Section II, the spectra are truncated at some frequency $\pm \Delta$. There are the following types of spectral profiles: i) flat-top (solid curve in Fig. 9), ii) convex (solid curve in Fig. 8 and dotted curve in Fig. 9), iii) finger-like (dotted curve in Fig. 8) and iv) concave (dashed curves in Figs. 89). All these types are widely presented in laser experiments and numerical simulations [11, 12, 13, 15, 16, 21].

One can see, that, as a rule, the CDS spectra relating to the + branch (Fig. 8) are broader 


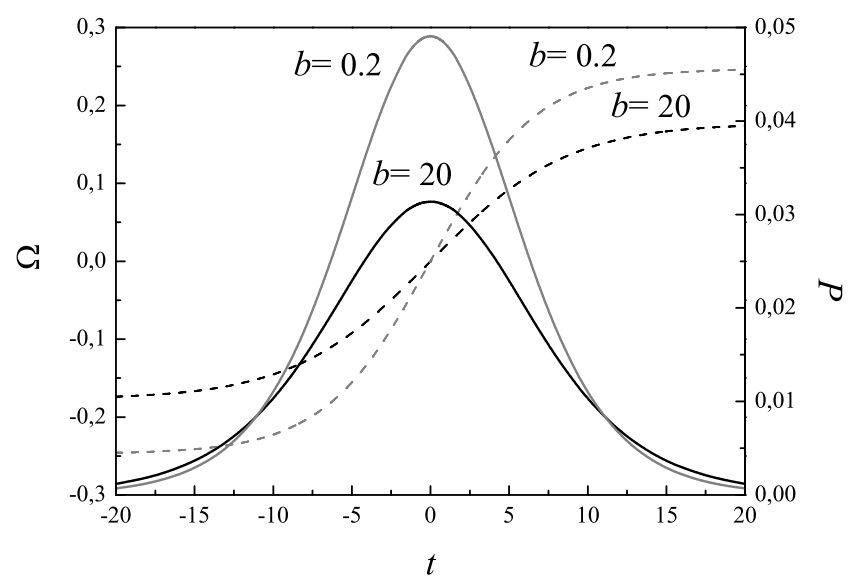

FIG. 7: The - branch CDS profiles (solid curves) and frequency deviations (dashed curves) for the different $b . c=1, a=0.01$.

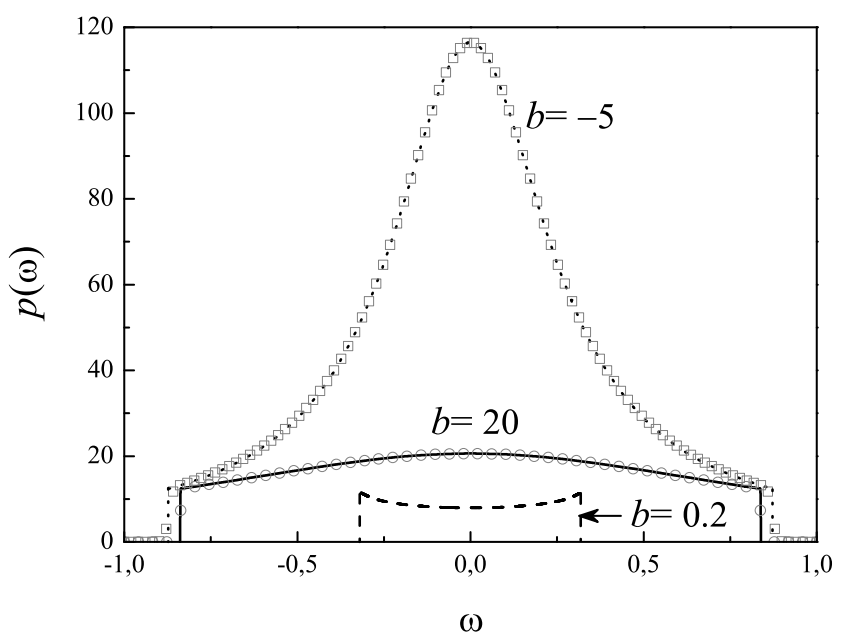

FIG. 8: The + branch CDS spectra for the different $b . c=1, a=0.01$. Solid, dotted and dashed curves correspond to analytical spectra. Gray circles correspond to the numerical spectrum at the point $\beta$ in Fig. $3\left(\zeta=0.5 \gamma, \beta / \alpha=25, \kappa=0.04 \gamma, E=820 \kappa \sqrt{\kappa \zeta} / \gamma^{2}\right)$. Gray squares correspond to the numerical spectrum at the point $\zeta$ in Fig. 5 ( $\zeta=0.5 \gamma, \beta / \alpha=25, \kappa=0.04 \gamma, E=2350$ $\left.\kappa \sqrt{\kappa \zeta} / \gamma^{2}\right)$.

than those relating to the - branch (Fig. 9). The cause of this difference is a smaller chirp for the - branch CDS. The spectrum narrows (widens) with an approach of positive $b$ to zero for the $+(-)$ branch in accordance with a decrease (increase) of the CDS chirp. When the positive quintic SPM increases $(b \rightarrow 0)$, concave spectra appear. In contrast to the model of [22], the source of such spectra is not the self-amplifying SAM [i.e., the negative $\zeta$ in Eq. 


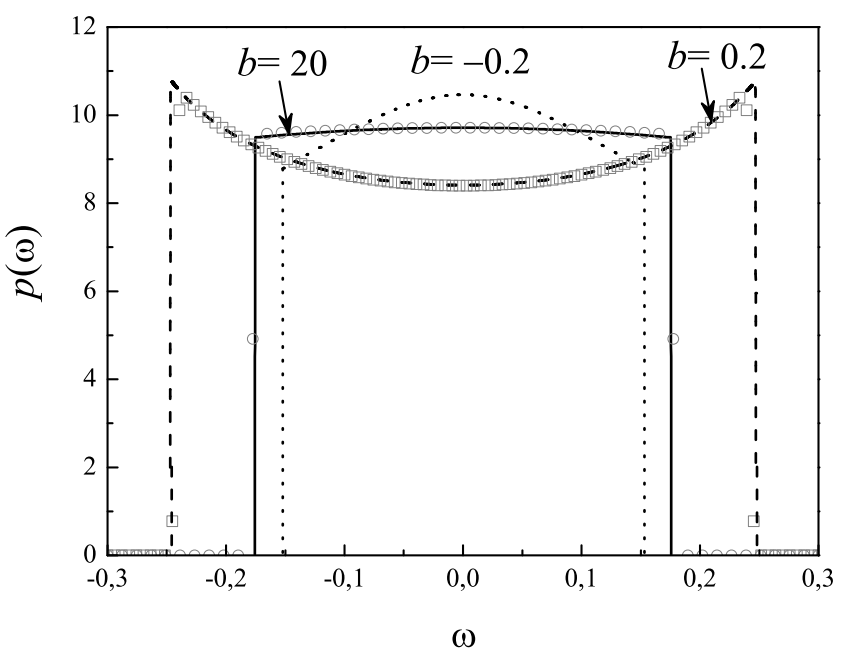

FIG. 9: The - branch CDS spectra for the different b. $c=1, a=0.01$. Solid, dotted and dashed curves correspond to the analytical spectra. Gray circles correspond to the numerical spectra at the point $\alpha$ in Fig. 3 (two coinciding numerical profiles are defined by $\zeta=0.1 \gamma ; \beta / \alpha=30$ and $40 ; \kappa=0.033 \gamma$ and $0.025 \gamma ; E=280 \kappa \sqrt{\kappa \zeta} / \gamma^{2}$ and $E=430 \kappa \sqrt{\kappa \zeta} / \gamma^{2}$, respectively). Gray squares correspond to the numerical spectrum at the point $\gamma$ in Fig. 4 ( $\zeta=0.1 \gamma, \beta / \alpha=42, \kappa=0.024 \gamma$, $\left.E=600 \kappa \sqrt{\kappa \zeta} / \gamma^{2}\right)$.

(1)] but solely the positive quintic SPM [24]. As a result, the concave spectrum solution of Section 2 is stable against collapse.

It is important to note, that a verging of $b$ towards zero for the + branch as well as a transition to the - branch reduce chirp. This can violate a validity of the method of stationary phase (see Section [II). As a result, the spectrum edges become smooth (see Section $\mathrm{V})$.

As was mentioned earlier, the + branch does not vanish along the curve of $\sigma=0$. This curve corresponds to marginal stability against a vacuum excitation and the CDS has a broadest spectrum here. The dependence of half-width of such a spectrum on the $c$ parameter for the different $b$ are shown in Fig. 10. In the absence of the quintic SPM $(b \gg 1)$, the dependence is symmetric relatively $c=1$, where the spectral width is maximum. The maximum $\Delta$ lowers (rises) and shifts towards $c=0(c=2)$ for the positive (negative) $b \rightarrow 0$ (Fig. 10). When $b>-4.5$, the + branch disappears for $a=0$ and $c=1$.

Fig. 11 demonstrates the dependencies of the spectral half-width on $a$ for a varied $b$ and a fixed $c$. As a result of larger energy and chirp, the + branch (solid curves) has a larger 


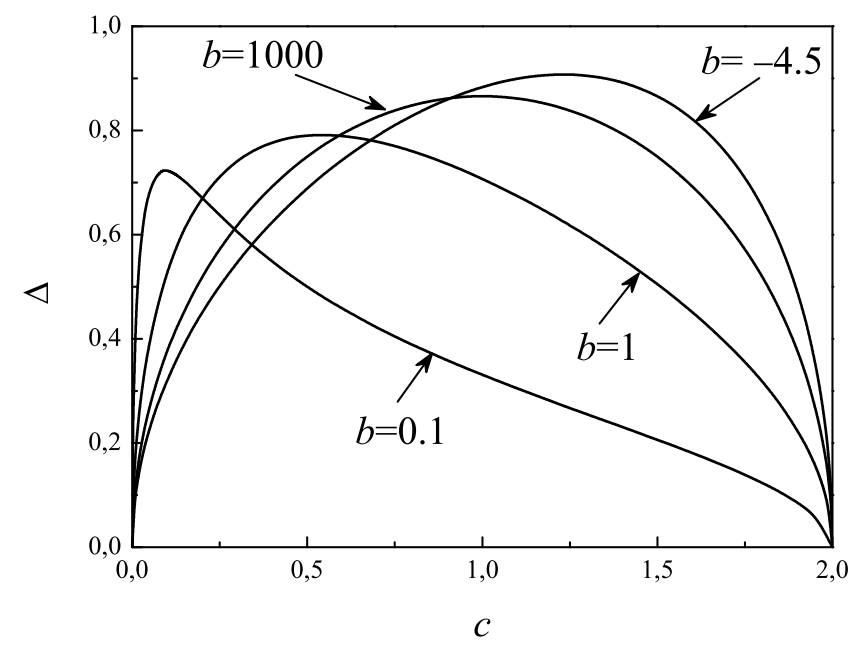

FIG. 10: The CDS spectral half-widths for the + branch and the different $b . a=0$.

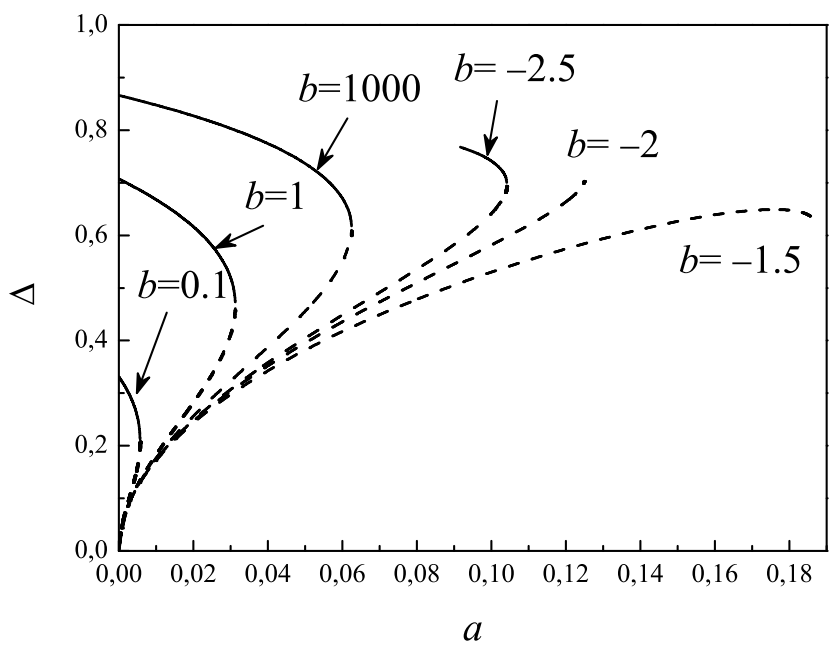

FIG. 11: The CDS spectral half-widths for the + (solid curves) and - (dashed curves) branches in dependence on $a$ for the different $b$ and $c=1$.

spectral width, which decreases with $a$ and the positive $b$ verging towards zero. The region of the + branch existence shortens with $b \rightarrow 0$ (also, see Fig. 1). A negative $b$ expands the + branch region towards a larger $a$. However, such a region is disconnected with $a=0$, if $b>-4.5$ for $c=1$ (see the region for $b=-2.5$ in Fig. 1). The existence of this minimum $c$ providing the CDS with $a=0$ is a result of asymptotical behavior of the zero isogain in Fig. 5. Physically, absence of the limit $a \rightarrow 0$ can mean that such a CDS is not able to develop from the vacuum of Eq. (11). Fig. 11 demonstrates that the + branch disappears completely, when $b \rightarrow-2$. 
The - branch has a lower spectral width, which increases with $a$ (Fig. 11, dashed curves). There exists some maximum $a$ (for a fixed $c$ ), where both branches merge.

\section{NUMERICAL SIMULATION OF THE CDS}

The above obtained approximate solution for the CDS has to be verified numerically. With this purpose, a symmetrized split-step Fourier method is used for numerical solving of Eq. (11). The temporal greed contains $2^{16}$ points, and the nonlinear propagation is simulated in the time domain using a fourth-order Runge-Kutta method. Total propagation distance consists of $\geq 10^{4}$ steps.

The simulations demonstrate, that the necessary factor providing the CDS stability is a dependence of $\sigma$ on $E$. Such a dependence is chosen to be in the form presented in Section III (the $\delta$ parameter equals to 0.5$)$ [15]. Then, the $E$ parameter in a master diagram can be easily replaced by the $E^{*}$ one, but the difference between $E$ and $E^{*}$ is small and, therefore, insignificant for further consideration.

The simulated spectra of the CDS are shown by open circles and squares in Figs. 8]9 for the parameters $a, b$ and $c$ corresponding to the points $\alpha, \beta, \gamma$ and $\zeta$ in Figs. 3]4,5. The agreement between the analytical and numerical results is perfect. Moreover, the numerical results demonstrate that the CDS is really three-parametric and its parameters scale in accordance with the rules of Section II. This means that the normalized parameters and profiles of the CDSs are identical for the identical sets of $(a, b, c)$. For instance, two parametric sets: i) $b=20, a=0.01, \beta / \alpha=30, \zeta=0.1 \gamma, E^{*}=280 \kappa \sqrt{\kappa \zeta} / \gamma^{2}, \kappa=0.033 \gamma$ [e.g., a $100 \mathrm{~nJ}$ Ti:sapphire oscillator with $\alpha=2.5 \mathrm{fs}^{2}$ and $\gamma=4.55 \mathrm{MW}^{-1}$ [15]]; and ii) $\beta / \alpha=40$, $E^{*}=430 \kappa \sqrt{\kappa \zeta} / \gamma^{2}, \kappa=0.025 \gamma$ correspond to the single point $\alpha$ in Fig. 3. This is the - branch, and the analytical (solid curve) as well as numerical (gray open circles) profiles coincide in Fig. 9, A single difference between the numerical and analytical spectra is that the former ones have gently smoothed edges. One has note, that a scalability of the CDS resembles the property of a true soliton, which is a solution with not fixed parameters [4].

The numerically obtained stability borders are shown in Figs. 34,5] by open squares. The stability condition is $\sigma>0$, that provides a vacuum stability. One can see, that both analytical and numerical borders coincide.

It is of interest to compare the analytical results with the numerical ones presented in 


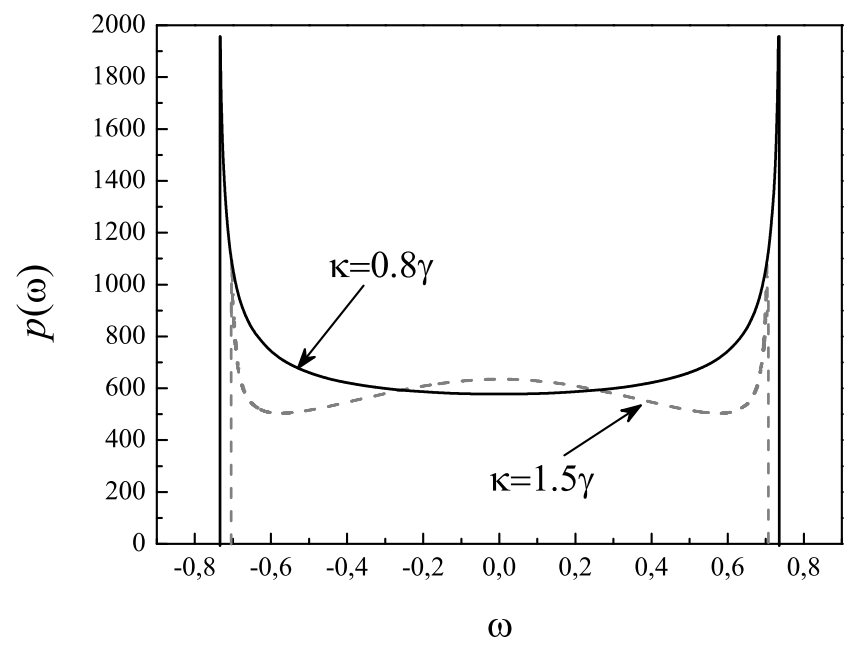

FIG. 12: The analytical CDS spectra for the + branch at the points $\delta$ ( $\kappa=0.8 \gamma$; black solid curve) and $\varepsilon(\kappa=1.5 \gamma$; gray dashed curve $)$ in Fig. 4. $b=0.2, \beta / \alpha=6.25, \zeta=0.002 \gamma$, and $\sigma=0.1$.

[10, 12]. There is a difference between the parametric sectors considered in [10, 12] and in this work. The case of $\beta \rightarrow 0$ lies beyond a validity of the analytical model under consideration, which requires $\beta \gg \alpha$. If $\beta$ approaches $\alpha$ and then tends to zero (as well as if $\kappa$ prevails over $\gamma$ ), the spectrum edges become smooth [12, 16] rather than truncated. The scaling rules of Section II and the requirement of $c<2$ can get broken in this case [25].

Nevertheless, i) strong scalability of $E$ with $\beta$, as well as both ii) existence of maximum $\beta$ and iii) minimum $\kappa$ providing a stable soliton suggest that the solutions analyzed in [10, 12] belong to the + branch (here we consider only normal dispersions). In conformity with [10], the stable CDS exists within the region of normal dispersion $(\beta>0)$ for both positive and negative $\chi$ (Figs. 1,2). A fast disappearance of the CDS with the increase of $b<0[10$ ] is the characteristic feature of the + branch (Fig. 1).

As expected, the CDS of [12] belongs to the + branch (the points $\delta$ and $\varepsilon$ in Fig. 41). The corresponding analytical spectra are shown in Fig. $12[p(\omega)$ for the black solid curve is re-scaled for convenience]. Both analytical spectra match with the numerical ones in Fig. 3 of [12] with the exception of the smoothed edges for the latter owing to $\kappa \geq \gamma$. Such a smoothing enhances for the - branch (the point $\epsilon^{\prime}$ in Fig. 4) because a chirp is lower for this branch.

When $\kappa<\gamma$, the spectrum is concave (black solid curve in Fig. 12) like that in Fig. 8 for $b=0.2$. When $\kappa$ exceeds $\gamma$, the new type of a spectral shape appears: the concave-convex 
one (gray dashed curve in Fig. 12). Such spectra have been studied numerically in [12] for $\kappa>\gamma$.

\section{CONCLUSION}

In conclusion, approximate chirped solitary pulse solutions of the cubic-quintic nonlinear CGLE have been constructed analytically under condition of domination of a dispersion over a spectral dissipation. The solutions are three-parametric and easily traceable within a whole parametric space, which has been represented in the form of the master diagrams. The solutions are divided into two branches, which differ in their energies and scaling properties. It is found, that the chirped dissipative solitons under consideration have truncated spectra with the concave, convex and concave-convex tops. Numerical analysis and comparisons with the existing results have demonstrated, that the approximate analytical solutions are stable and highly-accurate. The obtained results are of interest, in particular, for a development of both solid-state and fiber laser oscillators aimed to a generation of femtosecond pulses with over-microjoule energy.

\section{Acknowledgments}

Author thanks Boris Malomed for pointing out the solutions of [6, 7]. This work was supported by the Austrian Fonds zur Förderung der wissenschaftlichen Forschung (FWF project P20293).

[1] I.S.Aranson, L.Kramer, Rev. Mod. Phys. 74, 99 (2002).

[2] N.N.Akhmediev, A.Ankiewicz, Solitons: Nonlinear Pulses and Beams (London: Chapman and Hall, 1997).

[3] F.X.Kärtner (Ed.), Few-cycle Laser Pulse Generation and its Applications (Berlin: Springer Verlag, 2004).

[4] N.N.Akhmediev, A.Ankiewicz, (Eds.), Dissipative Solitons (Berlin, Heidelberg: Springer Verlag, 2005). 
[5] R. Conte, (Ed.), The Painlevé Property. One Century Later (New York: Springer Verlag, 1999).

[6] B.A.Malomed, Physica D 29, 155 (1987).

[7] B.A.Malomed, A.A.Nepomnyashchy, Phys. Rev. A 42, 6009 (1990).

[8] N.N.Akhmediev, A.Ankiewicz, (Eds.), Dissipative Solitons: From Optics to Biology and Medicine (Berlin, Heidelberg: Springer Verlag, 2008).

[9] H.A.Haus, J.G.Fujimoto, E.P.Ippen, J. Opt. Soc. Am. B 8, 2068 (1991).

[10] J.M.Soto-Crespo, N.N.Akhmediev, V.V.Afanasjev, S.Wabnitz, Phys. Rev. E 55, 4783 (1997).

[11] V.L.Kalashnikov, E.Podivilov, A.Chernykh, S.Naumov, A.Fernandez, R.Graf, Apolonski, New Journal of Physics 7, 217 (2005).

[12] N.Akhmediev, J.M.Soto-Crespo, Ph.Grelu, Physics Letters A 372, 3124 (2008).

[13] S.Naumov, A.Fernandez, R.Graf, P.Dombi, F.Krausz, A.Apolonski, New J. Phys. 7, 216 (2005).

[14] G.Palmer, M.Schultze, M.Siegel, M.Emons, U.Bünting, U.Morgner, Opt. Lett. 33, 1608 (2008).

[15] V.L.Kalashnikov, E.Podivilov, A.Chernykh, A.Apolonski, Appl. Phys. B: Lasers Opt. 83, 503 (2006).

[16] V.L.Kalashnikov, A. Apolonski, Phys. Rev. A 79, 043829 (2009).

[17] V.L.Kalashnikov, A.Fernández, A.Apolonski, Optics Express 16, 4206 (2008).

[18] B.Proctor, E.Westwig, F.Wise, Optics Lett. 18, 1654 (1993).

[19] A.Biswas, S.Konar, Introduction to non-Kerr Law Optical Solitons (Boca-Raton: Chapman\& Hall, 2007).

[20] E.Podivilov, V.L.Kalashnikov, JETP Lett. 82, 524 (2005).

[21] A.Chong, W.H.Ronninger, F.W.Wise, J. Opt. Soc. Am. B 25, 140 (2008).

[22] W.H.Ronninger, A.Chong, F.W.Wise, Phys. Rev. A 77, 023814 (2008).

[23] V.L.Kalashnikov, Maple worksheet (unpublished) http://info.tuwien.ac.at/kalashnikov/NCGLE2.html

[24] V.L.Kalashnikov, Proc. SPIE 7354, Nonlinear Optics and Applications III, Mario Bertolotti, (Ed.), $73540 \mathrm{~T}(2009)$.

[25] perturbative analysis of the limit of $\alpha, \gamma, \chi \rightarrow 0$ is presented in [7], where the soliton-like kink-antikink solutions of Eq. (1) have been found. 